\title{
Underappreciated features of cultural evolution
}

\author{
Marco Smolla ${ }^{1, *}$, Fredrik Jansson ${ }^{2,3}$, Laurent Lehmann ${ }^{4}$, Wybo Houkes ${ }^{5}$, Franjo \\ Weissing ${ }^{6,7}$, Peter Hammerstein ${ }^{8}$, Sasha R. X. Dall ${ }^{9}$, Bram Kuijper ${ }^{9}$, and Magnus \\ Enquist ${ }^{2,10}$ \\ ${ }^{1}$ Department of Biology, University of Pennsylvania, Philadelphia, PA, USA \\ ${ }^{2}$ Centre for Cultural Evolution, Stockholm University, Stockholm, Sweden \\ ${ }^{3}$ Division of Applied Mathematics, Mälardalen University, Västerås, Sweden \\ ${ }^{4}$ Department of Ecology and Evolution, University of Lausanne, Biophore, Lausanne, Switzerland \\ ${ }^{5}$ Philosophy and Ethics, Eindhoven University of Technology, Eindhoven, The Netherlands \\ ${ }^{6}$ Groningen Institute for Evolutionary Life Sciences, University of Groningen, Groningen, The \\ Netherlands \\ ${ }^{7}$ Netherlands Institute for Advanced Study, Amsterdam, The Netherlands \\ ${ }^{8}$ Institute for Theoretical Biology, Humboldt University Berlin, Germany \\ ${ }^{9}$ Centre for Ecology and Conservation, University of Exeter, Penryn Campus, Penryn, Cornwall, United \\ Kingdom \\ ${ }^{10}$ Department of Zoology, Stockholm University, Stockholm, Sweden \\ *Email: smolla@sas.upenn.edu
}

\begin{abstract}
Cultural evolution theory has long been inspired by evolutionary biology. Conceptual analogies between biological and cultural evolution have led to the adoption of a range of formal theoretical approaches from population dynamics and genetics. However, this has resulted in a research programme with a strong focus on cultural transmission. Here, we contrast biological with cultural evolution, and highlight aspects of cultural evolution that have not received sufficient attention previously. We outline possible implications for evolutionary dynamics and argue that not taking them into account will limit our understanding of cultural systems. We propose twelve key questions for future research, among which are calls to improve our understanding of the combinatorial properties of cultural innovation, and the role of development and life history in cultural dynamics. Finally, we discuss how this vibrant research field can make progress by embracing its multidisciplinary nature.
\end{abstract}

Keywords: cultural evolution, evolutionary biology, human evolution, cumulative culture, cultural systems 


\section{Introduction}

Culture is one of the most complex collective phenomena we know. The capacity of our species to produce and accumulate culture (i.e., the collection of socially transmitted attitudes, beliefs, knowledge, skills, technologies, values, etc.) has allowed us to inhabit virtually every part of the planet, making culture a cornerstone to the ecological success of our species (e.g. Henrich, 2015; van Schaik, 2016). Moreover, no other species produces as many different and complex cultural products and is undoubtedly as reliant on them as we are (Boyd and Richerson, 1995; van Schaik, 2016). As such, the study of culture and how it changes has been central to the research programme of many disciplines, from the humanities and social sciences to the natural sciences.

To better understand the origin and nature of culture, how culture emerges and changes over time, why some aspects of culture change continuously or abruptly while others remain relatively unchanged, or why sometimes neighbouring groups differ and distant groups share certain cultural practices, there has been a centuries-long effort to devise or formulate a general theory of culture. One such approach is the application of evolutionary theory to culture. Inspired by Darwin's publication 'On the Origin of Species' (1859), the idea of taking an evolutionary perspective on (human) culture has received considerable research interest from scholars in many fields. The popularity of using evolutionary terminology when describing cultural change, however, has varied considerably over time (for overviews of evolutionary thinking in the humanities and social sciences, see e.g. Carneiro, 2003; Gintis, 2007; Hodgson, 2019; Laland and Brown, 2011; Munro, 1963; Sanderson, 1990). This was in part due

to a lack of understanding of biological evolution but also based on the politically motivated misuse of evolutionary theory (see Mesoudi, 2011).

A more recent approach to studying cultural evolution was inspired by the quantitative theories that emerged in the life sciences in the first half of the twentieth century. They were the direct result of major advances in the understanding of the evolutionary process, marked by the Modern Synthesis that unified Darwinian evolution with Mendelian genetics. These novel formal descriptions of biological transmission greatly increased the understanding of biological evolution. The theoretical models by Fisher (1930), Wright (1931), and Haldane (1932) cast verbal theories and thought experiments in the precise language of mathematics, which allowed a systematic analysis of the processes involved in and the consequences for biological evolution. Moreover, using formal theoretical models has proven useful for extracting the most important features from complex processes, by keeping track of many often parallel processes, and explain complex macroscopic patterns (e.g. diversity and distribution of traits) with simpler individual-level processes, such as variability, competition, and inheritance.

Inspired by this progress, the now seminal works by Cavalli-Sforza and Feldman (1981), Lumsden and Wilson (1981), and Boyd and Richerson (1985) aimed collectively at a general theory of culture. The idea was to base this new theory on mathematical approaches from population dynamics and genetics to explain the distribution and change of traits in populations of individuals, be it genetic or cultural traits. To justify this approach, the authors 
assumed and outlined structural and conceptual analogies between biological and cultural evolution. At an abstract level, population-level processes in both domains are characterised by the continuous emergence, selective retention, and transmission of variation (Campbell, 1965; Jablonka and Lamb, 2014; Lewontin, 1970; Maynard Smith and Szathmáry, 1995). Hence, analogies have been made between the forces driving the evolution of genetic traits (transmission, mutation, drift, and selection) and those driving the evolution of cultural traits (transmission, innovation, random fluctuations, and selection). Additionally, both types are characterised by observable phenomena (e.g. an adapted species and cumulative culture) that are high-level properties (of a species and a population respectively), which are the result of low-level interactions (at the level of individuals). Finally, both biological and cultural evolution are cumulative processes. That is, traits evolve successively through repeatedly modifying, combining, re-combining, and re-purposing existing traits. These novel traits could not evolve in just one or a few generations due to their historical dependencies (Buskell et al., 2019). This process can lead to adaptation and increased trait complexity. However, the latter is not strictly necessary for the former as adaptations can sometimes come with a significant reduction in complexity (Szathmáry and Maynard Smith, 1997). In Box 1 we summarise the most important similarities which are discussed more thoroughly elsewhere (Boyd and Richerson, 1985; Campbell, 1965; Cavalli-Sforza and Feldman, 1981; Jablonka and Lamb, 2014; Mesoudi, 2007, 2017; Nelson, 2007; Szathmáry and Maynard Smith, 1997).

In contrast to the aforementioned general similarities, there are obvious differences between cultural and biological evolution that require mathematical approaches to be adapted (Creanza et al., 2017). For example, if parents exhibit different variants of a cultural trait (e.g. hunting versus fishing), then there is no general principle that makes their children prefer one over the other. Children might even reject traits from both parents, which is impossible for genetic traits. Additionally, cultural traits are not strictly acquired from parents (vertical transmission). Instead, transmission can occur between any individuals, from one-to-many or many-to-one. And so, traits can also be acquired from peers (horizontal) or other adults (oblique), even inverse transmission from younger to older individuals is common. Moreover, biases towards copying successful, powerful, prestigious, or otherwise notable individuals can further modify the transmission dynamics (Henrich and McElreath, 2003). The different transmission modes and biases, and their combinations, give rise to a large and complex space of potential patterns and dynamics in the distribution of cultural traits.

In fact, this space is so rich that the overwhelming amount of cultural evolution research has primarily focused on cultural transmission. What the aforementioned seminal books started has become a rich and vibrant research field that has garnered much interest from researchers across disciplines, leading to a large number of recent books (e.g. Acerbi, 2019; Henrich, 2015; Heyes, 2018; Laland, 2017; Mesoudi, 2011) and themed journal issues (e.g. Gelfand and Kashima, 2015; Kolodny et al., 2018; Whiten et al., 2017, 2011). Moreover, recent research articles show that the study of cultural transmission is not only relevant for understanding what shaped human origins, but also to make sense of what is happening in the present, from the spread of news on social media (Weng et al., 2013) to healthy behaviour 
in social networks (Centola, 2010), and many other features of the 'information age'. This development has also sparked the question to what extent culture exists in other animals (van Schaik, 2016), given the growing evidence for wide-spread social learning in nature (Aplin, 2019; Hoppitt and Laland, 2013; Krützen et al., 2005; Leadbeater and Chittka, 2007; Rendell and Whitehead, 2001; Whiten, 2019).

Precisely because of this surge of interest, we believe it is an ideal moment to identify potential avenues for future cultural evolution research. We believe that there are a number of differences between biological and cultural evolution that have still not received sufficient attention. We identify specific differences by contrasting characteristics of biological and cultural evolution and point out some (Table 1) that are of special interest. Our analysis mainly focuses on unique features of the acquisition, storage, recall, and transmission, and the emergence and selection of new cultural traits and how they differ from those associated with genetically inherited traits. We then discuss the consequences of these differences and potential implications for our understanding of cultural evolution. Our main aim is to contribute to opening up new lines of investigation, novel insights, and testable hypotheses.

This paper is an attempt to elucidate and discuss important similarities and differences between biological and cultural evolution. We are aware that there are many different ways to approach this task, and so this list will never be exhaustive. Additionally, we constrain our scope to modelling approaches that rely on individual interactions to explain the emergence of higher level patterns, as compared to those that, for example, apply phylogenetic methods on observed patterns to infer past dynamics (see e.g. Atkinson and Gray, 2005; Mace and Holden, 2005). However, we believe that this discourse provides interesting, even provocative, new material for thought and discussion, which, we hope, will inform future research, experimental testing and especially formal theoretical work.

Box 1. General similarities between biological and cultural evolution.

I. Evolution takes place in populations of individuals or units

II. Continuous generation of new variants based on existing traits

III. Differential retention of existing traits

IV. Transmission of traits between individuals

V. Evolution is the outcome of many microscopic events (II, III, and IV)

VI. Outcomes and emerging patterns:

- Diversity and divergence

- Cumulative evolution

- Historical dependencies

- Complexity and adaptation 


\section{Differences between biological and cultural evolution}

In this section, we summarise several important differences between biological and cultural evolution, before we discuss implications for theories of cultural evolution in the following section. Table 1 is an attempt to identify core differences by contrasting various aspects of biological and cultural evolution. Some of the listed differences have been considered previously (Boyd and Richerson, 1985; Cavalli-Sforza and Feldman, 1981; Creanza et al., 2017; Mesoudi, 2011; Whiten et al., 2017) while others have received less attention. We argue that recognising these differences will affect the understanding of cultural evolution in general, and modelling approaches in particular, as it is well known that even small changes to the mechanisms of dynamical systems can fundamentally change their behaviour, results, and interpretation.

It is important to note that (human) cognitive abilities, many unique, are a factor in many of the items listed under cultural evolution in the table and thus have consequences for the dynamics and outcome of cultural evolution. For instance, opportunities for cultural recombination and thus generation of new culture is significantly enhanced by human thinking, planning, and problem-solving abilities. These abilities will also play an important role in individual filtering as well as in learning processes.

The table entries would look partly different, for example, if we considered bacteria (with access to horizontal gene transfer (Gogarten and Townsend, 2005)), and cultural artefacts and externalised information storage (see section 2.1). One aspect that is not included in the table is processing. While it is undoubtedly integral to both biological and cultural evolution, some sort of processing is occurring in all items of the table.

\subsection{Storage}

The way in which information is stored differs fundamentally between cultural and biological evolution. In biological evolution, information (genetic traits) is encoded in and stored as the sequence of physical molecular structures, the genome. In contrast, in cultural evolution, cultural information within individuals (cultural traits) takes the form of mental representations, which are encoded as neuronal connections in the brain. This difference in the nature of trait storage means that an individual cannot change its genome, but can make changes to the composition of its cultural traits. For example, while genes can be frequently and accurately accessed and copied, there are very limited possibilities of passing information to the genome that can be inherited by offspring. In contrast, mental representations cannot be directly observed or copied (Tamariz, 2019), but due to the high flexibility of the nervous system, mental representations can be continuously altered before they are exhibited and transmitted to others. These differences have consequences for the evolutionary dynamics and concerns several of the other items in the table, which we will address in section 3.

Since artefacts (cultural products) reside in the external world, all the information for producing them often needs not be contained within individuals and can be easily trans- 
Table 1. A list of differences between biological and cultural evolution. The table is organised in a sequence of logical steps, beginning with the storage, acquisition, transmission and selection of traits, and ending with the generation of new variants. To keep the table comprehensive but intuitive, we used general models of evolution, with a standard model of a sexually reproducing species in the biological evolution column, and a minimal case of human cultural evolution in which culture is predominately stored as mental representations in the cultural evolution column.

\begin{tabular}{|cll}
\hline Trait & Biological evolution & Cultural evolution \\
\hline Storage & & \\
\hline \multicolumn{1}{c}{ Medium } & Genome & Nervous system \\
\hline Requisition & Recall only & Recall and alteration \\
\hline Transmission & Single event at conception & Sequential events throughout life \\
\hline \multicolumn{1}{|c|}{ Mechanism } & Transmission of physical copies & $\begin{array}{l}\text { Learning processes based on } \\
\text { social observations and } \\
\text { communication as input }\end{array}$ \\
\hline Pathways & From parents to offspring & Between any individuals \\
\hline Receiver dependence & No & Yes \\
\hline $\begin{array}{l}\text { Sorting processes } \\
\text { variants }\end{array}$ & Natural selection & $\begin{array}{l}\text { Highly diverse and often in } \\
\text { combination with other processes }\end{array}$ \\
\hline
\end{tabular}

mitted and accumulated over generations. Further, in the light of our ability to externalise information storage, computation, and filtering (and in part creation), an increasingly important question is how information technology changes cultural evolution as well as our mental abilities (see e.g. Acerbi, 2019).

\subsection{Acquisition}

Based on the difference in the nature of trait storage in cultural and biological evolution, the acquisition of traits is also fundamentally different. Genetic traits are in place at the beginning of an individual's life, whereas cultural traits are acquired throughout the entire 
life. An important consequence of continuous acquisition is that culture is acquired sequentially through a combination of episodes of social learning (learning from others), and individual practice (trial-and-error, insight, etc.). The importance of individual practice cannot be understated. It has been demonstrated in numerous studies in developmental psychology and educational science that many cultural traits require substantial individual efforts to be successfully learned (see e.g. Bjorklund and Causey, 2017; Hattie, 2008). For example, hunting skills require extensive social learning to acquire the fundamental behaviours and techniques but they also rely on lifelong improvement through individual learning (LewLevy et al., 2017). This difference between cultural and biological evolution has received rather limited attention, but it may be one of the most important ones influencing evolutionary dynamics (Enquist et al., 2010; Strimling et al., 2009). As we will discuss below, the fact that humans are born without culture and acquire culture sequentially, making the individual an active force in the transmission, has several consequences for the dynamics of cultural evolution, including, for instance, the fact that previously acquired traits can facilitate or even preclude the subsequent acquisition of other traits, and that an individual may transmit different trait variants during different stages of its life. The fact that culture is acquired sequentially does not exclude the importance of inborn predispositions or abilities in the acquisition of culture.

\subsection{Transmission}

Mechanism In contrast to the transmission of physical copies of instructions in biological systems, in cultural systems transmission happens indirectly through learning processes based on observations of other individuals' behaviours, or their products (including communication, objects, or alterations to the environment), such that the observer can form its own mental representation of a cultural trait. This difference between cultural and biological evolution is widely recognised (Boyd and Richerson, 1985; Cavalli-Sforza and Feldman, 1981; Mesoudi, 2007) and consistent with general findings in developmental psychology and educational science (see e.g. Bjorklund and Causey, 2017; Hattie, 2008). Nevertheless, the concrete transmission process and its consequences for the evolutionary dynamics are not fully understood and are subject to an ongoing debate (see Section 3.2). At its centre is the role of the relative fidelity of transmission in cultural dynamics. Two main schools of thought have emerged. The "California" school sees accurate transmission as the main cause for cultural stability (Acerbi and Mesoudi, 2015; Boyd and Richerson, 1985; Cavalli-Sforza and Feldman, 1981; Henrich and Boyd, 2002), whereas the "Paris" school highlights the importance of constant reconstruction through biased transformation towards cultural attractors (Claidière et al., 2014; Morin, 2016; Sperber, 1996). Recent attempts to to unify these two schools rely on differentiating between cultural traits that can be faithfully copied, that is, actions, and those that cannot, for example beliefs and ideas (Tamariz, 2019).

It is also important to note the importance of language in the transmission of cultural traits (Dean et al., 2012; Maynard Smith and Szathmáry, 1995; Pinker, 2015; Smith and 
Kirby, 2008). It allows for the transmission of complex data by replacing direct observations, delivering insightful synthetic information, and providing effective feedback (Morgan et al., 2015). There are likely many cultural traits that require language communication to be successfully transmitted, for example, cognitive abilities such as counting, reading, and mental arithmetic.

Pathways Given that mental representations of cultural traits are transmitted through observation and recreation, there is no fixed transmission direction. Compared to biological evolution where genes are transmitted from parents to offspring, transmission in cultural evolution can occur between any individuals. The diversity of possible transmission pathways, which in themselves are subject to cultural evolution, leads to interesting complex patterns.

Receiver dependence Another interesting and increasingly recognised difference of transmission in biological and cultural evolution is that genetic traits are supplied all at once, without the participation of the new individual. In contrast, in the transmission of cultural traits, not only the sender (teacher, model, or demonstrator) but also the receiver (student, or observer) can actively affect the transmission dynamics and thus evolutionary dynamics (Boyd and Richerson, 1985; Lumsden and Wilson, 1981). For instance, the observer may be selective about what and from whom to learn (Boyd and Richerson, 1985; Heyes, 2016; Lumsden and Wilson, 1981). Such biases or social learning strategies (Kendal et al., 2005; Laland, 2004) may be inborn (Boyd and Richerson, 1985; Lumsden and Wilson, 1981) or stem from previously acquired cultural traits (Buskell et al., 2019, Jansson et al., this issue).

\subsection{Sorting processes}

Sorting processes are processes that lead to a non-random change in the frequency of traits. In biological evolution, the main sorting process is natural selection. How it operates is usually clear and well understood, and it brings a general understanding of biological adaptions. In cultural evolution, the situation is considerably more complex, which is in part due to the active role each individual takes in acquiring, exhibiting, and transmitting cultural traits (Boyd and Richerson, 1985). The most explored sorting mechanism that involves the individual in this way, operates in the receiver during social learning, in which content, model or conformity biases favour the acquisition of certain traits while rejecting others (Richerson and Boyd, 2005). A trait may also be favoured or rejected subsequently when individuals get experiences of using the trait (Jansson et al., nd, this issue). Sorting will also occur if individuals selectively display traits to potential learners, and when the generation of new trait variants is incentive-driven. Individuals may also exercise social control by for instance trying to influence what others do or communicate by selectively punishing or rewarding such acts (Fehr and Gächter, 2002; Ostrom et al., 1992; Yamagishi, 1986). In addition, there is natural selection in cultural evolution, since cultural traits can influence survival and reproduction of their carriers and so affect trait frequencies (Boyd and Richerson, 1985; Cavalli-Sforza and 
Feldman, 1981). For instance, a habit that increases survival may spread because it will make carriers of the trait more common among demonstrators. For the same reason, a habit that decreases survival will gradually disappear.

In summary, sorting is possibly the most complex part of the evolutionary dynamics of cultural evolution, as it involves a number of different processes, and several of these occur in combination with other key processes such as transmission and generation of new culture.

\subsection{Generation of variation}

The continuous generation of variation is a central aspect of evolution. A generally recognised difference between biological and cultural evolution is that genetic variation is random and unrelated to any need or purpose. In contrast, in cultural evolution the generation of new variants can also be incentive-driven. This means that it is directed towards solving a concrete problem or reaching a certain goal. Important mechanisms include cognitive processing (Kahneman, 2011), deliberate experimentation (Basalla, 1988; Mesoudi, 2008; Simonton, 1995), as well as trial-and-error and associative learning processes (Kahneman, 2011; Öhman et al., 2000). However, cultural variants may also occur because of mistakes, transmission errors, by-products of trying to solve other problems or other circumstances that produce results that the individuals may learn from and subsequently transmit to other individuals (Basalla, 1988). Note that successful learning from unintended or unexpected outcomes is not free from incentive-driven aspects as it requires that the individual recognises how such observations can be used.

\section{Implications and challenges for cultural evolution theory}

The differences that we discussed in the previous section can have dramatic effects on the evolutionary dynamics. In this part, we will discuss a selection of these that we think are particularly important to the further development of cultural evolution theory.

\subsection{Potential for fast evolution and complex culture}

Cultural evolution can proceed much faster than biological evolution both in rate of change and in terms of trait complexity. This has been pointed out previously, and several reasons are generally given for why this is the case (Boyd and Richerson, 1985; Cavalli-Sforza and Feldman, 1981; Henrich, 2015; Mesoudi, 2011; Perreault et al., 2012; Richerson and Boyd, 2005; Shennan, 2002). Firstly, acquisition and transmission of cultural traits occur along many pathways and throughout an individual's entire lifetime. Secondly, novel or modified traits can be transmitted directly to other individuals (which is why cultural evolution is sometimes described as Lamarckian rather than Darwinian). Thirdly, the generation of novel variants can be incentive-driven. This may have a dramatic effect on the rate of evolution, since cultural traits will be much more likely to be productive once they 'enter' the real world. 
And finally, language can be an important tool for efficient cultural transmission. It is necessary for transmitting abstract ideas, beliefs and values, and a tool for the transmission of complex cultural traits, such as opaque processes, inaccessible observations, practices, algorithms and other mental skills.

A full understanding of evolutionary rates and complex culture also requires a detailed understanding of cumulative cultural evolution. Cumulative culture is not only the accumulation of traits but also the innovation of new traits by combining or recombining existing traits (Enquist et al., 2011; Kolodny et al., 2015; Lewis and Laland, 2012). This can result in a gradual increase in complexity as combinations can be recombined continuously. This may offer a better theory of how cultural complexity and cultural systems emerge (see section 3.4). Also, it could explain, through the power of combinatorics, how cultural evolution can generate a never-ending stream of new variation.

For a better understanding of the role of language and recombination in cumulative cultural evolution, future research needs to address several important questions, such as:

1. What are the combinatorial properties that allow a seemingly unbounded generation of diversity and complexity in human cumulative cultural evolution?

\section{What is the role of language, symbolism, and cognitive abilities in cumulative cultural evolution?}

3. Can all types of cultural change be understood in evolutionary terms (listed in Table 1), or do we need additional theoretical frameworks?

\subsection{Developmental processes are directly involved in acquisition and trans- mission}

Development here refers broadly to the processes that shape and modify the individual during its lifetime. In biological evolution, development is usually separated from the processes that transmit genetic traits and its evolution is studied in life-history theory, which emphasises the role of trade-offs (Stearns, 1992). In contrast, in cultural evolution, development and transmission processes tend to be more intertwined as they occur on the same time scale. On the one hand, an individual's experience is shaped by sequential encounters with its surrounding cultural environment. On the other hand, the individual itself contributes to the makeup of that cultural environment.

Relevant progress in our understanding of developmental processes in humans has been made in a variety of disciplines, such as developmental psychology, educational science and linguistics (Bjorklund and Causey, 2017; Hattie, 2008). Several general findings from empirical research in these fields are relevant to cultural evolution. First and foremost, development is generally incremental and time-consuming, starting with learning simple features and elements that are later integrated into a functional practical or mental skill. This is obvious for advanced skills like mental arithmetic (Carey, 2011; Dehaene, 2009; Wynn, 1992), and 
reading and writing, but also required for more basic skills (see e.g. Carey, 2011; Friedman and Scholnick, 2014; Nelson and Gruendel, 1981).

A second key finding is that traits are typically acquired through a combination of social interactions and individual efforts (Bjorklund and Causey, 2017; Hattie, 2008; Nelson, 1999; Vygotsky, 1978). Thus, social and individual learning are operating together rather than being alternative strategies. Further, research on development has also clearly established that there is an extensive behavioural and mental flexibility in humans. This allows humans to acquire a large range of skills, many of recent origin and not part of our prehistory, like reading and writing, agricultural practices, playing chess, and computer programming. Performance is generally strongly correlated to the amount of training both for domain-specific and domain-general skills (Ericsson et al., 2006). Thus, psychology and educational science suggest that when we see faithful copying over generations, this depends on substantial learning efforts among individuals. The situation is complicated by findings showing that learning and memory abilities themselves respond to training, and are thus partly cultural and subject to cultural evolution (Bjorklund and Causey, 2017; Ericsson et al., 2006; Gobet and Charness, 2018).

Previous theoretical models of cultural evolution typically use simplified assumptions about individual development and rely on simple copying mechanisms and strategies for social learning (Enquist et al., 2007; Rendell et al., 2010a,b; Smolla et al., 2015), or treat social and individual learning as opposing alternatives (Boyd and Richerson, 1985; Rogers, 1988), whose evolution is generally studied in the absence of a learning-reproduction trade-off (see Wakano and Miura, 2014, for how classical results are critically changed once the learningreproduction trade-off is taken into account).

It is thus becoming increasingly clear that future models should incorporate developmental processes, especially for traits that cannot be acquired in a single learning episode (because they are combinations of simpler traits or because they require individual improvement). These models should further be embedded into a proper life-history context so as to make all traits pertaining to learning co-evolutionary (Kaplan et al., 2000).

Challenges and questions for future research:

1. How can developmental psychology and related fields inform cultural evolution research? For example, in the debate between the "California" and the "Paris" school on why cultural learning can be faithful to its source and maintained over generations, while single transmission events are error-prone (Acerbi and Mesoudi, 2015; Buskell, 2017; Henrich and Boyd, 2002; Sperber and Hirschfeld, 2004), developmental psychology might explain faithful transmission in terms of sequential learning including efficient corrective feedback loops.

2. What is the scope of cultural evolution in terms of mental abilities? To what extent are mental abilities and psychological mechanisms learnt from or influenced by the cultural environment (Elman et al., 1998; Karmiloff-Smith, 1994), and thus subject to 
cultural evolution themselves (Heyes, 2018)? Future models that combine developmental processes and cultural evolution may help to distinguish the roles of inborn factors, acquired traits, and individual experience.

\section{How does learning depend on an individual's lifetime and how has it co-evolved with life history?}

\subsection{Different evolutionary outcomes and cultural fitness}

In biological evolution, the fate of a trait can be understood by considering the average reproductive success of all carriers of that trait (Haig, 2012; Hamilton, 1963). It is common to use optimisation and game theoretic approaches to study evolutionary endpoints (i.e. adaptations). In contrast, in cultural evolution there is no generally accepted formulation of cultural fitness (Ramsey and De Block, 2017) as the relationship between the individual and its traits is more complicated. Here, transmission is not tied to biological reproduction, can occur between arbitrary individuals, and the individual is not fixed with respect to the cultural traits it possesses and exhibits to others during its life. These differences have implications both for the outcome of evolution and for how cultural evolution should be theoretically explored (Boyd and Richerson, 1985; Cavalli-Sforza and Feldman, 1981; Mesoudi, 2011; Strimling et al., 2009). In principle, anything that influences transmission, retention, and use of cultural traits needs to be considered.

For instance, traits that are contagious enough can spread and be maintained in a population even if they reduce the survival and/or biological reproduction of individuals (Anderton et al., 1987; Boyd and Richerson, 1976; Campbell, 1975). Suppose lifestyle $A$ produces more children than lifestyle $B$ and that parental lifestyles are often transmitted to the children. However, children are also exposed to the alternative lifestyle $B$, which is more attractive (e.g. because it is easier to learn). This may be driven by a genetic or cultural bias for this lifestyle. This could result in $B$ increasing in frequency compared to $A$. Figure 1 provides a numerical example. A complete analysis of cultural evolution scenarios of this kind requires exploration of both the evolution of lifestyles and the evolution of preferences for lifestyles (Kolk et al., 2014).

In any human society, we find a range of both material and non-material culture serving the well-being, survival and reproduction of individuals or the group. However, we can also observe phenomena that are more difficult to interpret as adaptive in this way. We spend significant amounts of time learning to play musical instruments and chess. Often, our actions and beliefs do not even promote the goals they are aimed at, like when anti-vaxxers refuse medically motivated vaccinations in order to promote health (Dubé et al., 2013).

It is quite possible that concepts like adaptation and fitness in cultural evolution will not have equivalent and as straightforward meanings as they do in biological evolution. The complication is that individuals are subject to change during their lives, which can be illustrated by comparing a trait that transmits rather slowly to others, but tends to stay with an 


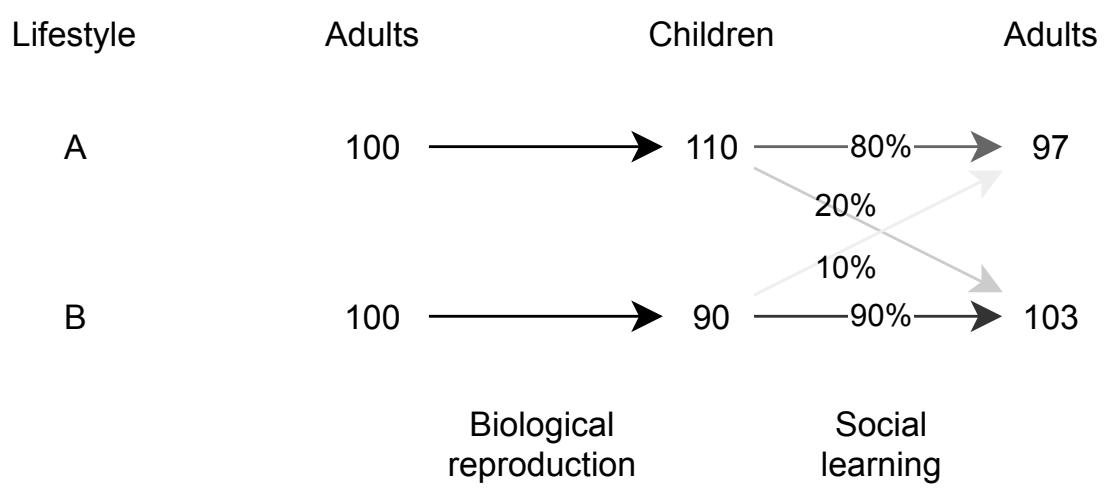

Figure 1. A numerical example showing how a lifestyle associated with lower fertility could spread in cultural evolution (see section 3.3).

individual once it is acquired, with a trait that transmits quickly to other individuals, but is easily abandoned or replaced. The latter trait may be easy to learn, or has some immediate attraction, but is less productive. Which trait spreads can depend on trait content and thus may not be predictable by considering an elementary set of rules of transmissions (Claidière and André, 2012).

It has been suggested that the fitness of cultural traits can be based on just the traits or free-floating memes (Ramsey and De Block, 2017). While this can be a useful approach for certain questions, a general theory of cultural evolution cannot ignore the individual because of its active roll in acquiring, processing, and exhibiting cultural traits (Ramsey and De Block, 2017). This is one of the reasons that make it so tricky to find a useful concept of units of evolution in cultural evolution (a unit might be the single cultural trait or a set of traits, but also the carrier of a trait, etc.).

Challenges and questions for future research:

1. Is it possible to provide a general theoretical account for the observed diversity of outcomes of human cultural evolution?

2. How can we define and recognise cultural adaptations, at what level are they manifested, and how can we use them to identify useful units of cultural evolution?

3. What could be a useful concept of fitness in cultural evolution, and, if it exists, how can it be used to study cultural evolution? 


\subsection{Trait interactions, cultural systems, and evolving evolutionary dynam- ics}

The facts that individuals acquire traits sequentially and that traits can interact may have important organising effects. The resulting developmental path dependence (see Section 3.2) can limit which traits an individual acquires, and so promote the evolution of cultural systems (Buskell et al., 2019; Claidière et al., 2014; Goldberg and Stein, 2018; Yeh et al., 2019, Jansson et al. this issue). For instance, an individual might acquire a belief $A$ about the world, which subsequently facilitates the acquisition of other beliefs that are consistent with $A$ but leads to the rejection of beliefs that are not (Festinger, 1957). If, instead, the individual would first acquire an alternative belief $B$, then this could trigger the eventual acquisition of an entirely different belief system. Similar reasoning can apply to other categories of traits. In fact, there are many ways in which traits can interact and influence evolution. Traits may or may not operate well together (within or between individuals), they might express preferences towards other traits, and define goals the individual strives for that are better served by certain traits than others. Traits can also be clustered in space (e.g. co-occurrence of traits relevant for fishing near bodies of water) or time (seasonal occurrence of sets of traits), and may even influence social network structure, and thus lead to exposure to different sets of traits (Smolla and Akçay, 2019). The fact that culture exists in the form of systems, contexts or structures of interrelated parts, manifested both in individuals and in groups of individuals, is generally recognised in the humanities and social sciences (Buskell et al., 2019). It is possible that many cultural phenomena can only be understood from an explicit systems perspective.

Trait interactions could also have even more pronounced effects, and bring about changes to the evolutionary process itself, that is, important aspects of cultural evolution dynamics may themselves be results of cultural evolution rather than determined by genetic factors or external circumstances (Acerbi et al., 2014; Buskell et al., 2019; Kolodny et al., 2016). Examples of such changes in biological evolution are migration, mutation and sexual reproduction, which are called modifier traits. These traits have important consequences for the dynamics of biological evolution, but they are also products of that same evolution, thus resulting in counterintuitive feedbacks (Altenberg and Feldman, 1987). All items in Table 1 are potentially subject to cultural evolution, and it has been suggested that cultural evolution itself can influence social learning abilities (Henrich, 2015), efforts by senders' and receivers' willingness to change (Acerbi et al., 2014; Ghirlanda et al., 2006), and transmission pathways (Smolla and Akçay, 2019). Culturally established goals could have a dramatic effect on the outcome of cultural evolution. Acerbi et al. (2014) introduced the term regulatory traits for cultural traits that influence the dynamics of the evolutionary process and suggested that their evolution depends on their ability to directly or indirectly influence their own evolution.

The organising effects of interactions and feedbacks can also be seen in the broader perspective of evolutionary transitions. These are fundamental modifications to the evolutionary process, caused by evolution itself. Maynard Smith and Szathmáry (1995) recognised several properties common to such transitions, which we can also find in human cultural evolution. 
The first property is that new ways of transmitting information are formed. Humans have invented a series of information technologies, like reading and writing, radio and television, databases, and pocket calculators, which can store, as well as communicate or broadcast cultural traits, and also help us think and make decisions. A second property of transitions is that smaller entities aggregate to form larger ones and that the smaller entities tend to differentiate. Humans form a diversity of societies that are conceivably different from those that existed at the dawn of human culture, with for instance complex political and social organisations, division of labour, individual specialisations and long production chains, and organised transmission of cultural traits via institutions like schools (see e.g. Carneiro, 2003; Richerson et al., 2016; Scarre, 2005; Turchin, 2016).

Challenges and questions for future research:

1. What new understanding can be gained from a systems approach that cannot be gained from considerations of simpler representations of culture?

2. How can the evolution of cultural systems be explored theoretically? This requires formal descriptions of cultural systems, an understanding of trait relationships, and evolutionary modelling approaches that take into account that cultural systems exist and operate within and between individuals (Jansson et al., nd, this issue).

\section{How do externalised information storage and processing affect cultural evolu- tion?}

\section{Concluding remarks}

Studying cultural evolution in an evolutionary framework has come a long way. The development of this research programme has proven invaluable to our understanding of many cultural phenomena. The many differences between cultural and biological evolution, while not invalidating an evolutionary approach, show that there is a rich research field with many potentially interesting avenues for future studies. The way mental representations are stored, recalled, transmitted, and modified, and the active role the individual plays in all of these stages, leads to a level of complexity that may even require entirely new analytical and modelling approaches to be fully understood.

We hope that the collection of ideas we have presented here have highlighted some of the complexity of cultural evolution, which occurs at different organisational levels, along different timelines, between the material and the immaterial, and the biotic and abiotic world. Clearly, a full understanding of culture requires interdisciplinary efforts. Fortunately, cultural evolution research has always been at home in a variety of disciplines. And on closer inspection, much work has been evolutionary at its core, even if the term was not explicitly used.

The discussions at the Leiden meeting have emphasised the value of overcoming semantic barriers that exist across disciplines. Despite different historical motivations, perspectives, 
and approaches to cultural evolution research, there are shared ambitions and overlapping questions. We would like to close this essay with three suggestions to strengthen the interdisciplinary approach and to counteract isolation between different schools of thought.

Focusing on questions relevant to the social sciences and humanities To strengthen interdisciplinary work, we believe it would be useful to address more of the cultural and social phenomena that are intensively studied within the humanities and the social sciences. Potential examples are tribalism, race and gender issues, knowledge resistance, segregation, social immobility, systems of belief, cooperation, trust and polarisation. There has been a tendency to focus on general questions in cultural evolution modelling, such as the emergence, change, transmission, and accumulation of cultural traits. By considering more specific phenomena, it should also be possible to generate new concrete and testable hypothesis. Perhaps the best example of a productive interaction between cultural evolutionary theorising and empirical research, blending general and specific theories with in-depth knowledge about the phenomena, can be found in recent developments in language evolution (Croft, 2000).

Acknowledging existing work in other fields We also think that it is important to pay more attention to existing thinking and theory within the humanities and the social sciences, and also to make use of the wealth of existing empirical data on human culture. There are two aspects to this. One is that evolutionary thinking is not a novel concept in the humanities and social sciences as we discussed above. The other is that many theories, including verbal, statistical and mathematical models, are genuinely evolutionary in our sense without using the term evolution. Thus there are clear overlapping goals with cultural evolutionary research. This applies to both older verbal hypotheses and recent theoretical work. A few examples of the latter include: studies on social learning dynamics in evolutionary game theory in economics (Sandholm, 2011; Schlag, 1998); the dynamics of knowledge production and acquisition at the aggregate (population) level studied in macroeconomics (Galor and Weil, 2000; Reiter, 2012; Romer, 1990, 1994); and models of beliefs of agents studied in sociology (DeGroot, 1974; Friedkin et al., 2016). Microeconomics has also adopted the cultural evolution framework, as introduced by Cavalli-Sforza and Feldman, and apply it to all sorts of questions (Bisin and Verdier, 2000, 2001). An important next step in acknowledging existing research must be synthetic work. It has become increasingly clear that it is almost impossible to embrace all theories of cultural change that exist. There is a real need for synthetic work, to avoid reinvention, and to recognise both general and specific theories about cultural change that deserve to be further explored.

Connecting disciplines Finally, we suggest making use of knowledge from and cooperation with a few fields that are rarely mentioned in formal theoretical studies of cultural evolution. Two such fields that we have mentioned throughout this paper are developmental psychology and educational science. Together these fields have gathered important insights 
into individual development and social learning. Many of the scenarios described, and the interaction with individual learning, differ from assumptions and strategic thinking about social learning common in cultural evolution research. They also offer insight into naturenurture issues, the scope of cultural evolution, and how mental abilities can be transmitted. Another field of potential interest is artificial intelligence and related fields in cognitive science, which hold many ideas about learning processes and cognitive abilities.

\section{Acknowledgement}

This paper is a summary of discussions at the "Foundations of Cultural Evolution" workshop in Leiden, Netherlands, in 2019, upon which this special issue is based. F.W. was one of the organisers. We also thank Pieter van den Berg, Eva Boon and Lucas Molleman for organising the workshop and inviting us.

\section{Funding}

M.S. was supported by an ARO grant to Erol Akçay (W911NF-17-1-0017). F.J. and M.E. were supported by grant 2015.0005 from the Knut and Alice Wallenberg Foundation.

\section{Author contributions}

M.S., F.J., and M.E. wrote the paper, and all authors contributed to discussions and revisions.

\section{Competing interests}

The authors declare that they have no competing interests.

\section{References}

Acerbi, A. (2019). Cultural Evolution in the Digital Age. Oxford University Press, Oxford, New York.

Acerbi, A., Ghirlanda, S., and Enquist, M. (2014). Regulatory Traits: Cultural Influences on Cultural Evolution. In Cagnoni, S., Mirolli, M., and Villani, editors, Evolution, Complexity and Artificial Life, pages 135-147. Springer Berlin Heidelberg, Berlin, Heidelberg.

Acerbi, A. and Mesoudi, A. (2015). If we are all cultural Darwinians what's the fuss about? Clarifying recent disagreements in the field of cultural evolution. Biology \& Philosophy, 30(4):481-503. 
Altenberg, L. and Feldman, M. W. (1987). Selection, generalized transmission and the evolution of modifier genes. I. The reduction principle. Genetics, 117(3):559-572.

Anderton, D. L., Tsuya, N. O., Bean, L. L., and Mineau, G. P. (1987). Intergenerational transmission of relative fertility and life course patterns. Demography, 24(4):467.

Aplin, L. M. (2019). Culture and cultural evolution in birds: A review of the evidence. Animal Behaviour, 147:179-187.

Atkinson, Q. D. and Gray, R. D. (2005). Curious parallels and curious connectionsphylogenetic thinking in biology and historical linguistics. Systematic biology, 54(4):51326.

Basalla, G. (1988). The Evolution of Technology. Cambridge University Press.

Bisin, A. and Verdier, T. (2000). A model of cultural transmission, voting and political ideology. European Journal of Political Economy, 16(1):5-29.

Bisin, A. and Verdier, T. (2001). The economics of cultural transmission and the dynamics of preferences. Journal of Economic Theory, 97(2):298-319.

Bjorklund, D. F. and Causey, K. B. (2017). Children's Thinking: Cognitive Development and Individual Differences. Sage Publications.

Boyd, R. and Richerson, P. J. (1976). A simple dual inheritance model of the conflict between social and biological evolution. Zygon, 11(3):254-262.

Boyd, R. and Richerson, P. J. (1985). Culture and the Evolutionary Process. University of Chicago Press, Chicago.

Boyd, R. and Richerson, P. J. (1995). Why culture is common, but cultural evolution is rare. Proceedings of the British Academy, 88:77-93.

Buskell, A. (2017). What are cultural attractors? Biology \& Philosophy, 32(3):377-394.

Buskell, A., Enquist, M., and Jansson, F. (2019). A systems approach to cultural evolution. Palgrave Communications, 5(1):131.

Campbell, D. T. (1965). Variation and selective retention in socio-cultural evolution. In Barringer, H. R., Blanksten, G. I., and Mack, R. W., editors, Social Change in Developing Areas: A Reinterpretation of Evolutionary Theory, pages 19-49. Schenkman, Cambridge, Massachusetts.

Campbell, D. T. (1975). On the conflicts between biological and social evolution and between psychology and moral tradition. American Psychologist, 30(12):1103-1126. 
Carey, S. (2011). The Origin of Concepts. Oxford University Press, Oxford; New York.

Carneiro, R. L. (2003). Evolutionism in Cultural Anthropology: A Critical History. Westview Press, Cambridge, MA.

Cavalli-Sforza, L. L. and Feldman, M. W. (1981). Cultural Transmission and Evolution: A Quantitative Approach. Number 16 in Monographs in Population Biology. Princeton University Press, Princeton, N.J.

Centola, D. (2010). The spread of behavior in an online social network experiment. Science, 329(5996):1194-1197.

Claidière, N. and André, J.-B. (2012). The Transmission of Genes and Culture: A Questionable Analogy. Evolutionary Biology, 39(1):12-24.

Claidière, N., Scott-Phillips, T. C., and Sperber, D. (2014). How Darwinian is cultural evolution? Philosophical transactions of the Royal Society of London. Series B, Biological sciences, 369(1642):20130368-20130368.

Creanza, N., Kolodny, O., and Feldman, M. W. (2017). Cultural evolutionary theory: How culture evolves and why it matters. Proceedings of the National Academy of Sciences, 114(30):7782-7789.

Croft, W. (2000). Explaining Language Change: An Evolutionary Approach. Longman Linguistics Library. Longman, Harlow, England ; New York.

Darwin, C. (1859). The Origin of Species by Means of Natural Selection. Reprinted by The Modern Library. Random House, New York.

Dean, L. G., Kendal, R. L., Schapiro, S. J., Thierry, B., and Laland, K. N. (2012). Identification of the social and cognitive processes underlying human cumulative culture. Science, 335(6072):1114-1118.

DeGroot, M. H. (1974). Reaching a consensus. Journal of the American Statistical Association, 69(345):118-121.

Dehaene, S. (2009). Origins of mathematical intuitions: The case of arithmetic. Annals of the New York Academy of Sciences, 1156(1):232-259.

Dubé, E., Laberge, C., Guay, M., Bramadat, P., Roy, R., and Bettinger, J. A. (2013). Vaccine hesitancy: An overview. Human Vaccines \& Immunotherapeutics, 9(8):1763-1773.

Elman, J. L., Bates, E. A., Johnson, M. H., Karmiloff-Smith, A., Plunkett, K., and Parisi, D. (1998). Rethinking Innateness: A Connectionist Perspective on Development, volume 10. The MIT Press. 
Enquist, M., Eriksson, K., and Ghirlanda, S. (2007). Critical social learning: A solution to Rogers's paradox of nonadaptive culture. American Anthropologist, 109(4):727-734.

Enquist, M., Ghirlanda, S., and Eriksson, K. (2011). Modelling the evolution and diversity of cumulative culture. Philosophical Transactions of the Royal Society B: Biological Sciences, 366(1563):412-423.

Enquist, M., Strimling, P., Eriksson, K., Laland, K., and Sjostrand, J. (2010). One cultural parent makes no culture. Animal Behaviour, 79(6):1353-1362.

Ericsson, K. A., Charness, N., Feltovich, P. J., and Hoffman, R. R., editors (2006). The Cambridge Handbook of Expertise and Expert Performance. Cambridge University Press, Cambridge ; New York.

Fehr, E. and Gächter, S. (2002). Altruistic punishment in humans. Nature, 415(6868):137140.

Festinger, L. (1957). A Theory of Cognitive Dissonance, volume 2. Stanford university press, Stanford.

Fisher, R. (1930). The Genetical Theory of Natural Selection. The Clarendon Press, United Kingdom.

Friedkin, N. E., Proskurnikov, A. V., Tempo, R., and Parsegov, S. E. (2016). Network science on belief system dynamics under logic constraints. Science, 354(6310):321-326.

Friedman, S. L. and Scholnick, E. K. (2014). The Developmental Psychology of Planning: Why, How, and When Do We Plan? Taylor and Francis, Hoboken.

Galor, O. and Weil, D. N. (2000). Population, Technology, and Growth: From Malthusian Stagnation to the Demographic Transition and Beyond. American Economic Review, 90(4):806-828.

Gelfand, M. J. and Kashima, Y. (2015). Culture: Advances in the science of culture and psychology. Current Opinion in Psychology, 8:1-7.

Ghirlanda, S., Enquist, M., and Nakamaru, M. (2006). Cultural evolution develops its own rules. Current Anthropology, 47(6):1027-1034.

Gintis, H. (2007). A framework for the unification of the behavioral sciences. Behavioral and Brain Sciences, 30(1):1-16.

Gobet, F. and Charness, N. (2018). Expertise in chess. In Ericsson, K. A., Hoffman, R. R., Kozbelt, A., and Williams, A. M., editors, The Cambridge Handbook of Expertise and Expert Performance, pages 597-615. Cambridge University Press, second edition. 
Gogarten, J. P. and Townsend, J. P. (2005). Horizontal gene transfer, genome innovation and evolution. Nature Reviews Microbiology, 3(9):679-687.

Goldberg, A. and Stein, S. K. (2018). Beyond social contagion: Associative diffusion and the emergence of cultural variation. American Sociological Review, 83(5):897-932.

Haig, D. (2012). The strategic gene. Biology \& Philosophy, 27(4):461-479.

Haldane, J. B. S. (1932). The Causes of Evolution. Longmans, London.

Hamilton, W. D. (1963). The Evolution of Altruistic Behavior. The American Naturalist, 97(896):354-356.

Hattie, J. (2008). Visible Learning: A Synthesis of over 800 Meta-Analyses Relating to Achievement. Taylor and Francis, London; New York, 1st edition.

Henrich, J. and Boyd, R. (2002). On Modeling Cognition and Culture: Why cultural evolution does not require replication of representations. Journal of Cognition and Culture, 2(2):87112.

Henrich, J. and McElreath, R. (2003). The evolution of cultural evolution. Evolutionary Anthropology: Issues, News, and Reviews, 12(3):123-135.

Henrich, J. P. (2015). The Secret of Our Success. Princeton University Press, Princeton.

Heyes, C. (2016). Who Knows? Metacognitive Social Learning Strategies. Trends in Cognitive Sciences, 20(3):204-213.

Heyes, C. M. (2018). Cognitive Gadgets: The Cultural Evolution of Thinking. The Belknap Press of Harvard University Press, Cambridge, Massachusetts.

Hodgson, G. M. (2019). Evolutionary Economics: Its Nature and Future. Elements in Evolutionary Economics. Cambridge University Press.

Hoppitt, W. and Laland, K. N. (2013). Social Learning: An Introduction to Mechanisms, Methods, and Models. Princeton University Press, Princeton.

Jablonka, E. and Lamb, M. J. (2014). Evolution in Four Dimensions, Revised Edition: Genetic, Epigenetic, Behavioral, and Symbolic Variation in the History of Life. MIT press.

Jansson, F., Aguilar, E., Acerbi, A., and Enquist, M. (n.d.). Modelling Cultural Systems and Selective Filters.

Kahneman, D. (2011). Thinking, Fast and Slow. Farrar, Straus and Giroux, New York, 1st edition. 
Kaplan, H., Hill, K., Lancaster, J., and Hurtado, A. M. (2000). A theory of human life history evolution: Diet, intelligence, and longevity. Evolutionary Anthropology: Issues, News, and Reviews, 9(4):30.

Karmiloff-Smith, B. A. (1994). Beyond Modularity: A Developmental Perspective on Cognitive Science. International Journal of Language \& Communication Disorders, 29(1):95-105.

Kendal, R. L., Coolen, I., van Bergen, Y., and Laland, K. N. (2005). Trade-offs in the adaptive use of social and asocial learning. Advances in the Study of Behavior, 35:333-379.

Kolk, M., Cownden, D., and Enquist, M. (2014). Correlations in fertility across generations: Can low fertility persist? Proceedings of the Royal Society B: Biological Sciences, 281(1779):20132561.

Kolodny, O., Creanza, N., and Feldman, M. W. (2015). Evolution in leaps: The punctuated accumulation and loss of cultural innovations. Proceedings of the National Academy of Sciences, 112(49):E6762-E6769.

Kolodny, O., Creanza, N., and Feldman, M. W. (2016). Game-changing innovations: How culture can change the parameters of its own evolution and induce abrupt cultural shifts. PLoS computational biology, 12(12):e1005302.

Kolodny, O., Feldman, M. W., and Creanza, N. (2018). Bridging cultural gaps: Interdisciplinary studies in human cultural evolution. Philosophical Transactions of the Royal Society B: Biological Sciences, 373(1743):20170413.

Krützen, M., Mann, J., Heithaus, M. R., Connor, R. C., Bejder, L., and Sherwin, W. B. (2005). Cultural transmission of tool use in bottlenose dolphins. Proceedings of the National Academy of Sciences of the United States of America, 102(25):8939-43.

Laland, K. N. (2004). Social learning strategies. Animal Learning \& Behavior, 32(1):4-14.

Laland, K. N. (2017). Darwin's Unfinished Symphony: How Culture Explains the Evolution of the Human Mind. Princeton University Press, Princeton, NJ.

Laland, K. N. and Brown, G. R. (2011). Sense and Nonsense: Evolutionary Perspectives on Human Behaviour. Oxford University Press, New York, second edition.

Leadbeater, E. and Chittka, L. (2007). Social learning in insects - From miniature brains to consensus building. Current Biology, 17(16):R703-R713.

Lew-Levy, S., Reckin, R., Lavi, N., Cristóbal-Azkarate, J., and Ellis-Davies, K. (2017). How Do Hunter-Gatherer Children Learn Subsistence Skills?: A Meta-Ethnographic Review. Human Nature, 28(4):367-394. 
Lewis, H. M. and Laland, K. N. (2012). Transmission fidelity is the key to the build-up of cumulative culture. Philosophical Transactions of the Royal Society B: Biological Sciences, 367(1599):2171-2180.

Lewontin, R. C. (1970). The Units of Selection. Annual Review of Ecology and Systematics, 1(1):1-18.

Lumsden, C. J. and Wilson, E. O. (1981). Genes, Mind, and Culture: The Coevolutionary Process. Harvard University Press, Cambridge, Mass.

Mace, R. and Holden, C. (2005). A phylogenetic approach to cultural evolution. Trends in Ecology \& Evolution, 20(3):116-121.

Maynard Smith, J. and Szathmáry, E. (1995). The major evolutionary transitions. Nature, 374(6519):227-232.

Mesoudi, A. (2007). A Darwinian Theory of Cultural Evolution Can Promote an Evolutionary Synthesis for the Social Sciences. Biological Theory, 2(3):263-275.

Mesoudi, A. (2008). Foresight in cultural evolution. Biology \& Philosophy, 23(2):243-255.

Mesoudi, A. (2011). Cultural Evolution: How Darwinian Theory Can Explain Human Culture and Synthesize the Social Sciences. University of Chicago Press, Chicago.

Mesoudi, A. (2017). Pursuing Darwin's curious parallel: Prospects for a science of cultural evolution. Proceedings of the National Academy of Sciences, 114(30):7853-7860.

Morgan, T. J. H., Uomini, N. T., Rendell, L. E., Chouinard-Thuly, L., Street, S. E., Lewis, H. M., Cross, C. P., Evans, C., Kearney, R., de la Torre, I., Whiten, A., and Laland, K. N. (2015). Experimental evidence for the co-evolution of hominin tool-making teaching and language. Nature Communications, 6(1):6029.

Morin, O. (2016). Reasons to be fussy about cultural evolution. Biology and Philosophy, 31(3):447-458.

Munro, T. (1963). Evolution in the Arts and Other Theories of Culture History. The Journal of Aesthetics and Art Criticism, 22(1):75.

Nelson, K. (1999). Language in Cognitive Development: Emergence of the Mediated Mind. Cambridge Univ. Press, Cambridge.

Nelson, K. and Gruendel, J. (1981). Generalized event representations: Basic building blocks of cognitive development. In Lamb, M. E. and Brown, A. L., editors, Advances in Developmental Psychology, volume 1, pages 131-158. Erlbaum, Hillsdale, NJ. 
Nelson, R. R. (2007). Universal Darwinism and evolutionary social science. Biology \& Philosophy, 22(1):73-94.

Öhman, A., Lewis, M., and Haviland-Jones, J. M. (2000). Fear and anxiety: Evolutionary, cognitive, and clinical perspectives. In Handbook of Emotions, pages 573-93. The Guilford Press, New York.

Ostrom, E., Walker, J., and Gardner, R. (1992). Covenants with and without a Sword: SelfGovernance Is Possible. American Political Science Review, 86(2):404-417.

Perreault, C., Moya, C., and Boyd, R. (2012). A Bayesian approach to the evolution of social learning. Evolution and Human Behavior, 33(5):449-459.

Pinker, S. (2015). Language, Cognition, and Human Nature: Selected Articles. Oxford University Press, Oxford.

Ramsey, G. and De Block, A. (2017). Is Cultural Fitness Hopelessly Confused? The British Journal for the Philosophy of Science, 68(2):305-328.

Reiter, S. (2012). Knowledge, discovery and growth. In Olson, G. M., Malone, T. W., and Smith, J. B., editors, Coordination Theory and Collaboration Technology, pages 193-260. Lawrence Erlbaum Associates, Mahwah, NJ.

Rendell, L., Boyd, R., Cownden, D., Enquist, M., Eriksson, K., Feldman, M. W., Fogarty, L., Ghirlanda, S., Lillicrap, T., and Laland, K. N. (2010a). Why copy others? Insights from the social learning strategies tournament. Science, 328(5975):208-213.

Rendell, L., Fogarty, L., and Laland, K. N. (2010b). Rogers' paradox recast and resolved: Population structure and the evolution of social learning strategies. Evolution; international journal of organic evolution, 64(2):534-548.

Rendell, L. and Whitehead, H. (2001). Culture in whales and dolphins. Behavioral and brain sciences, 24:309-382.

Richerson, P., Baldini, R., Bell, A. V., Demps, K., Frost, K., Hillis, V., Mathew, S., Newton, E. K., Naar, N., Newson, L., Ross, C., Smaldino, P. E., Waring, T. M., and Zefferman, M. (2016). Cultural group selection plays an essential role in explaining human cooperation: A sketch of the evidence. Behavioral and Brain Sciences, 39.

Richerson, P. J. and Boyd, R. (2005). Not by Genes Alone: How Culture Transformed Human Evolution. University of Chicago Press, Chicago, Ill., 3rd edition.

Rogers, A. R. (1988). Does biology constrain culture. American Anthropologist, 90(4):819-831.

Romer, P. M. (1990). Endogenous Technological Change. Journal of Political Economy, 98(5):S71-S102. 
Romer, P. M. (1994). The Origins of Endogenous Growth. Journal of Economic Perspectives, $8(1): 3-22$.

Sanderson, S. K. (1990). Social Evolutionism: A Critical History. Studies in Social Discontinuity. Blackwell, Cambridge, Mass. USA.

Sandholm, W. H. (2011). Population Games and Evolutionary Dynamics. Economic Learning and Social Evolution. MIT Press, Cambridge, Mass.

Scarre, C., editor (2005). The Human Past: World Prehistory \& the Development of Human Societies. Thames \& Hudson, New York, N.Y, 1st ed edition.

Schlag, K. H. (1998). Why imitate, and if so, how? - A Boundedly Rational Approach to Multi-armed Bandits. Journal of Economic Theory, 78(1):130-156.

Shennan, S. (2002). Genes, Memes, and Human History: Darwinian Archaeology and Cultural Evolution. Thames \& Hudson, London.

Simonton, D. K. (1995). Foresight in insight? A Darwinian answer. In Sternberg, R. J. and Davidson, J. E., editors, The Nature of Insight, pages 465-494. The MIT Press.

Smith, K. and Kirby, S. (2008). Cultural evolution: Implications for understanding the human language faculty and its evolution. Philosophical Transactions of the Royal Society B: Biological Sciences, 363(1509):3591-3603.

Smolla, M. and Akçay, E. (2019). Cultural selection shapes network structure. Science Advances, 5(8):eaaw0609.

Smolla, M., Gilman, R. T., Galla, T., and Shultz, S. (2015). Competition for resources can explain patterns of social and individual learning in nature. Proceedings of the Royal Society B: Biological Sciences, 282(1815):20151405.

Sperber, D. (1996). Explaining Culture: A Naturalistic Approach. Blackwell, Oxford, UK ; Cambridge, Mass.

Sperber, D. and Hirschfeld, L. A. (2004). The cognitive foundations of cultural stability and diversity. Trends in Cognitive Sciences, 8(1):40-46.

Stearns, S. C. (1992). The Evolution of Life Histories. Oxford University Press, Oxford ; New York.

Strimling, P., Enquist, M., and Eriksson, K. (2009). Repeated learning makes cultural evolution unique. Proceedings of the National Academy of Sciences, 106(33):13870-13874.

Szathmáry, E. and Maynard Smith, J. (1997). From Replicators to Reproducers: The First Major Transitions Leading to Life. Journal of Theoretical Biology, 187(4):555-571. 
Tamariz, M. (2019). Replication and emergence in cultural transmission. Physics of Life Reviews, 30:47-71.

Turchin, P. (2016). Ultrasociety: How 10.000 Years of War Made Humans the Greatest Cooperators on Earth. Beresta Books, Chaplin, Connecticut.

van Schaik, C. P. (2016). The Primate Origins of Human Nature. Wiley, first edition.

Vygotsky, L. S. (1978). Mind in Society: The Development of Higher Psychological Processes. Harvard University Press, MA.

Wakano, J. Y. and Miura, C. (2014). Trade-off between learning and exploitation: The Paretooptimal versus evolutionarily stable learning schedule in cumulative cultural evolution. Theoretical Population Biology, 91:37-43.

Weng, L., Menczer, F., and Ahn, Y.-Y. (2013). Virality prediction and community structure in social networks. Scientific Reports, 3(1).

Whiten, A. (2019). Cultural Evolution in Animals. Annual Review of Ecology, Evolution, and Systematics, 50(1):1-22.

Whiten, A., Ayala, F. J., Feldman, M. W., and Laland, K. N. (2017). The extension of biology through culture. Proceedings of the National Academy of Sciences, 114(30):7775-7781.

Whiten, A., Hinde, R. A., Laland, K. N., and Stringer, C. B. (2011). Culture evolves. Philosophical Transactions of the Royal Society B: Biological Sciences, 366(1567):938-948.

Wright, S. (1931). Evolution in mendelian populations. Genetics, 16(2):97.

Wynn, K. (1992). Children's acquisition of the number words and the counting system. Cognitive Psychology, 24(2):220-251.

Yamagishi, T. (1986). The provision of a sanctioning system as a public good. Journal of Personality and Social Psychology, 51(1):110-116.

Yeh, D. J., Fogarty, L., and Kandler, A. (2019). Cultural linkage: The influence of package transmission on cultural dynamics. Proceedings of the Royal Society B: Biological Sciences, 286(1916):20191951. 